\title{
Emerging Viral Infections
}

Patterns of infectious disease transmission change with time. Human activities like land use changes, agriculture, travel and recreation, increased contacts with wild animal reservoirs, degradation of health care resources, intake of unusual food do play a role in the changing levels of risk. About 75\% of the emerging diseases are derived from animals. The zoonotic diseases spill over from their natural reservoirs either through direct contact or indirectly through close contact with domestic animals and subsequently into human populations. The most important event in new disease emergence is genetic changes in the pathogen that make it possible to become established in a new host species, infect new, typically humans and health threats.

\section{Influenza}

Human influenza usually refers to the influenza subtypes that spread widely in human population. H1N1, H1N2 and H3N2 are the known Influenza $A$ virus subtypes circulating among humans. The major animal reservoirs of Influenza $A$ are migratory birds and the majority of all the possible combinations of HA-NA subtypes have been isolated from birds. The first purely avian virus causing severe disease in humans was noted in 1997. The H5N1 virus spillover occurred to human poultry workers in China. The virus was a reassortant with the H5 derived from geese with the other genes apparently derived from viruses prevalent in quail from the same region. Direct transmission of avian influenza viruses of subtypes H7N7, H9N2, and H7N3 has been associated occasionally with human disease. Subsequent human cases have been linked with human exposure to poultry and poultry products. The increased prevalence of amantadine resistance in circulating human influenza is of growing concern.

\section{Adenovirus}

Adenovirus has been a sporadic cause of acute respiratory disease. An emerging serotype sometimes causes severe and occasionally fatal respiratory illness in patients of all ages. These cases are associated with varied clinical illness, including conjunctivitis, febrile upper respiratory illness, pneumonia and gastrointestinal manifestations. Severe illness can occur in newborn or elderly patients or in patients with underlying medical conditions. The control of adenovirus outbreaks is challenging because virus is shed in both respiratory secretions and feces and can persist for weeks on environmental surfaces.

\section{Human Polyomavirus}

In 2007, a previously unrecognized polyomavirus was identified from respiratory secretions in patents from various parts of the world. It indicated that infection with the viruses was geographically widespread, though at relatively low frequency. The extent of clinical disease appeared mild. Little is known about the epidemiology or methods of transmission of the viruses.

The most common clinical findings in the patients include cough, upper respiratory tract symptoms, tachypnea and infiltrates or consolidation on chest radiography. The most frequent diagnoses were pneumonia, bronchiolitis and upper respiratory tract infections.

\section{SARS-CoV}

Severe acute respiratory syndrome (SARS) appeared late in 2002 in southern China. Initial cases were recognized as atypical pneumonia. The etiologic agent (SARS-CoV) was identified as a previously unrecognized coronavirus.

\section{Chikungunya Virus}

Chikungunya virus was first isolated in 1952 from humans. This Alphavirus is considered to be transmitted principally by various members of the mosquito genus Aedes. The virus circulates within a cycle between mosquitoes and various wildlife species. Sporadic spillover to humans has been characterized by mostly small, localized outbreaks. A single genetic change may be responsible for the large increase in cases observed recently.

It presents with sudden onset of high fever, fatigue and disabling joint and muscle pain and may be associated with a maculopapular rash and gastrointestinal complaints. A mild hemorrhagic syndrome also has been described. Severe arthralgias may persist for up to six months, though mostly resolve within two months.

\section{West Nile Virus}

West Nile virus is a mosquito-borne, positive sense RNA virus that is transmitted between vertebrate animals and mosquitoes. Patterns of transmission may differ between urban and rural landscapes in the same region because of different susceptibilities of the mosquitoes and birds that occupy these habitats.

Transmission to humans is predominantly through mosquito bite. Most infected people are asymptomatic. Symptoms may develop in $20-40 \%$ of people with infection. Symptoms characterized by fever, headache, malaise, myalgia, fatigue, skin rash, lymphadenopathy, vomiting and diarrhea are usual features. A small fraction of cases develop severe neuroinvasive diseases like West Nile meningitis, West Nile encephalitis, and acute flaccid paralysis.

Jaydeep Choudhury Chairperson 